\title{
ROSAT PSPC/HRI observations of the open cluster NGC 2422
}

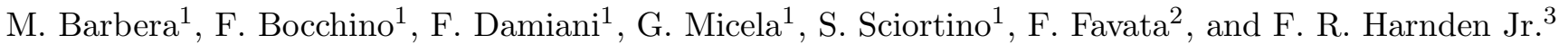 \\ 1 INAF - Osservatorio Astronomico di Palermo G.S. Vaiana, Piazza del Parlamento 1, 90134 Palermo, Italy \\ 2 Astrophysics Division, Space Science Department of ESA, ESTEC, Postbus 299, 2200 AG Noordwijk, \\ The Netherlands \\ 3 Harvard-Smithsonian Center for Astrophysics, 60 Garden St., Cambridge (MA), USA
}

Received 15 January 2001 / Accepted 14 February 2002

\begin{abstract}
We present the results of a ROSAT study of NGC 2422, a southern open cluster at a distance of about $470 \mathrm{pc}$, with an age close to the Pleiades. Source detection was performed on two observations, a 10-ks PSPC and a 40-ks HRI pointing, with a detection algorithm based on wavelet transforms, particularly suited to detecting faint sources in crowded fields. We have detected 78 sources, 13 of which were detected only with the HRI, and 37 detected only with the PSPC. For each source, we have computed the $0.2-2.0 \mathrm{keV}$ X-ray flux. Using optical data from the literature and our own low-dispersion spectroscopic observations, we find candidate optical counterparts for 62 X-ray sources, with more than $80 \%$ of these counterparts being late type stars. We have assigned to the optical sources an astrometric and/or a photometric membership flag, depending on which data are available to us. The number of sources (38 of 62) with high membership probability counterparts is consistent with that expected for Galactic plane observations at our sensitivity. We have computed maximum likelihood X-ray luminosity functions (XLF) for F and early-G type stars with high membership probability. Heavy data censoring due to our limited sensitivity permits determination of only the high-luminosity tails of the XLFs; the distributions are indistinguishable from those of the nearly coeval Pleiades cluster.
\end{abstract}

Key words. open clusters and associations: individual: NGC 2422 - stars: coronae - X-rays: stars

\section{Introduction}

The study of open clusters provides ideal samples of agehomogeneous solar-type (F, G and $\mathrm{K}$ ) stars that are essential to the understanding of the age evolution of stellar activity, as well as of the relation among coronal emission and other indicators of stellar activity. Among the better studied parameters related to stellar activity are the $\mathrm{X}$-ray luminosity, the projected rotation velocity $v \sin (i)$, the surface lithium abundance, and spectral indicators of enhanced chromospheric activity.

With most of the coeval stellar samples within $500 \mathrm{pc}$ now studied with ROSAT, a wide range of stellar parameters has already been explored. The ROSAT observations have largely confirmed the picture that emerged from early Einstein observations of a decaying X-ray luminosity level with increasing stellar age for $\mathrm{G}$ and $\mathrm{K}$ dwarfs in the range between $3 \times 10^{7}$ and $5 \times 10^{9}$ yr (Caillault 1996). Furthermore, these data allowed the determination of the X-ray luminosity level of $\mathrm{M}$ dwarfs in a few of these clusters (cf. Randich 1997). These results confirmed the

Send offprint requests to: M. Barbera,

e-mail: barbera@astropa.unipa.it evidence, already found in $\mathrm{G}$ and $\mathrm{K}$ stars, that the coronal X-ray emission evolution depends on stellar mass.

Some of the ROSAT observations have shown different age-activity relations for coeval stellar clusters (Jeffries 1999). For example, the average $\mathrm{X}$-ray luminosity of $\mathrm{G}$ and K stars in Praesepe appears to be much lower than in the Hyades in spite of Praesepe being in all aspects very similar to the Hyades from the optical point of view (Randich \& Schmitt 1995). Among possible explanations for this observational evidence are a different orbital period distribution of binaries (Barrado y Navascués et al. 1998), or the recent evidence for Praesepe being two merging clusters (Holland et al. 2000).

The X-ray study of the open cluster NGC 2422 allows us to consolidate and to test the current picture. With an estimate of its age of $8 \pm 2 \times 10^{7} \mathrm{yr}$ (van Rensbergen et al. 1978; Rojo Arellano et al. 1997), derived from Main Sequence (MS) fitting on standard UBV and Strömgren photometry and the use of theoretical isochrones, NGC 2422 is likely coeval or slightly younger than the Pleiades. Furthermore, its diameter $\left(\sim 30^{\prime}\right)$ matches well with the fields of view of the ROSAT Position Sensitive Proportional Counter (PSPC, Pfeffermann et al. 1987) and High Resolution Imager 
(HRI, Zombeck et al. 1995), and its reddening, $E(B-V) \sim$ 0.06 (Shobbrook 1984; Nissen 1988), is very small for its distance of $\sim 470$ pc (Rojo Arellano et al. 1997).

Various optical photometric studies of this cluster (with both photographic and photoelectric methods) are reported in the literature (cf. Zug 1933; Lyngå 1959; Hoag et al. 1961; Smyth \& Nandy 1962; Ishmukhamedov 1967), and more recently with the Strömgren photometry (Shobbrook 1984; Nissen 1988).

The distance modulus and reddening of the cluster have been measured by Shobbrook (1984) to be $m-M=$ 8.0 and $E(B-V)=0.06$, respectively, and by Nissen (1988) to be $m-M=8.14$ and $E(B-V)=0.053$, respectively. The Hipparcos measurement of the distance modulus, based only on four members of the cluster, is $m-M=8.48_{-0.42}^{+0.52}$ (Robichon et al. 1999).

The measurement of the metal abundance of the cluster conducted with Strömgren photometry does not provide evidence of significant difference between NGC 2422 and nearly coeval clusters like Pleiades and $\alpha$ Persei (Nissen 1988). Although it is well known that metallicity estimates from Strömgren photometry can be affected by activity in late type stars (cf. Morale et al. 1996; Favata et al. 1997), the metallicities reported in Nissen (1988) were derived with homogeneous criteria for the abovementioned clusters and based on the observation of $\mathrm{F}$ type stars where the metallicity determination from Strömgren photometry is less if at all sensitive to activity (Morale et al. 1996).

Our paper is organized as follows: in Sect. 2 we describe the X-ray data and analysis, in Sect. 3 we introduce the optical sample based on a catalog compiled from the literature and new observations we have carried out with the ESO $1.5 \mathrm{~m}$ telescope in La Silla. In Sect. 4 we discuss our main results.

\section{X-ray observations and data analysis}

NGC 2422 was observed with the PSPC and the HRI detectors on October 1993, and October/November 1994, respectively. The PSPC, which covers the $0.1-2.4 \mathrm{keV}$ energy range, has a circular field of view (FOV) of $\sim 2^{\circ}$ diameter and a spatial resolution of $\sim 40^{\prime \prime}$ full width at half maximum $(F W H M)$ on-axis and $\sim 2$ '.5 at $45^{\prime}$ off-axis. The PSPC spectral resolution is $E / \Delta E \sim 2$ at $1 \mathrm{keV}$. The HRI has smaller FOV $\left(36^{\prime} \times 36^{\prime}\right)$ and greater spatial resolution ( $\sim 6^{\prime \prime} F W H M$ on-axis), but no spectral resolution. The accumulated exposure times were 9 and $38 \mathrm{ks}$, for the PSPC and HRI, respectively. The two observations are offset by $2.7^{\prime}$, which is negligible compared to the respective fields of view, and are pointed toward the center of the cluster $\left(7^{\mathrm{h}} 36^{\mathrm{m}} 48^{\mathrm{s}},-14^{\circ} 25^{\prime} 48^{\prime \prime}\right.$, J2000.0).

\subsection{Source detection}

Time intervals highly contaminated by non-cosmic X-ray background have been removed using a time screening algorithm (Bocchino et al. 1998) optimized for the maximization of the signal to noise ratio of faint point sources. Both observations show low contamination and only a fraction of less than $1 \%$ of the total exposure time was discarded.

An algorithm based on wavelet transforms (Damiani et al. 1997a) was used for source detection and count rate determination on both the PSPC and HRI observations. This algorithm also calculates for each source the probability of existence above the detector background expressed in number of sigma of a Gaussian distribution (Damiani et al. 1997b, in the following significance of the source).

Sources detected with a significance greater than $4.0 \sigma$ (i.e. probability of existence greater than $99.99 \%$ ) were initially selected. With this threshold, the code is expected to detect $\sim 3$ spurious sources per field. We have detected 71 sources in the PSPC observation, and 45 sources in the HRI observation. The lists of the PSPC and HRI sources was then merged to create a single X-ray master catalog. The adopted positional matching error circle for the PSPC and HRI sources has been computed according to the analytical formulation derived by Damiani et al. $(1997 \mathrm{~b})$, i.e. $r_{90 \%}=0.525 \sigma_{\mathrm{psf}} \mathrm{e}^{5.433 / n_{\sigma}}$ where $\sigma_{\mathrm{psf}}$ is the width (standard deviation) of the Gaussian component of the PSF computed as a function of off-axis angle according to Hasinger et al. (1993) for the PSPC and David et al. (1995) for the HRI, and $n_{\sigma}$ is the source probability of existence expressed as the number of standard deviations of a Gaussian distribution. A further additional uncertainty of 10 arcsec has been added in quadrature to account for the known limitation of the absolute aspect reconstruction of the ROSAT satellite.

Figure 1 shows the $40^{\prime} \times 40^{\prime}$ central region of the PSPC $\mathrm{X}$-ray image with circles overlayed onto the positions of the detected sources, and Fig. 2 is the HRI X-ray image at the same scale with squares overlayed onto the positions of the HRI detected sources, and circles overlayed onto the positions of the PSPC detected sources with symbol size equal to the PSPC sources error circle.

From the X-ray sources detected only with one instrument, we have discarded those with significance less than 4.2 or $4.5 \sigma$, for the PSPC or HRI, respectively. Sources with lower significance were retained only if detected by both instruments. The adopted thresholds result in less than one spurious source in the field of view of each detector. The final X-ray catalog contains 28 HRI sources positionally matched with at least one PSPC source, 13 HRI sources with no PSPC counterpart and 37 PSPC sources with no HRI counterpart, for a total of 78 X-ray sources, reported in Table 1. We have used the notation $\mathrm{P} x x$ and $\mathrm{H} x x$, where $x x$ are two digits, for the PSPC-only and HRIonly X-ray sources, and four digits for sources detected both in PSPC and HRI, with the first two digits giving PSPC source number. 


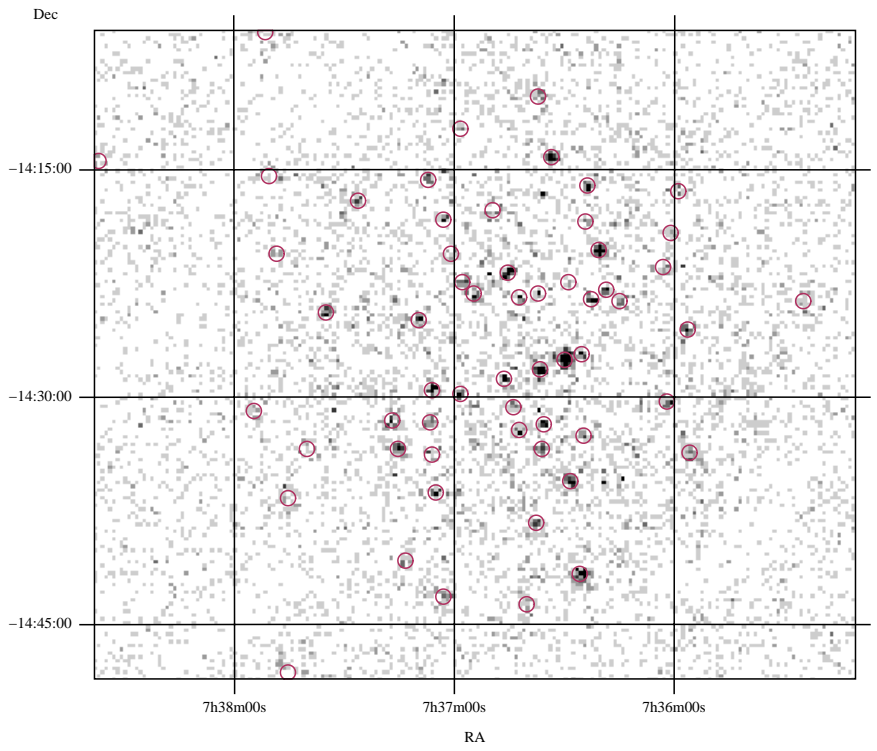

Fig. 1. Central $40^{\prime} \times 40^{\prime}$ region of the PSPC X-ray image of NGC 2422. X-ray sources detected with the wavelet transform algorithm in this $10 \mathrm{ks}$ observation are indicated with small circles.

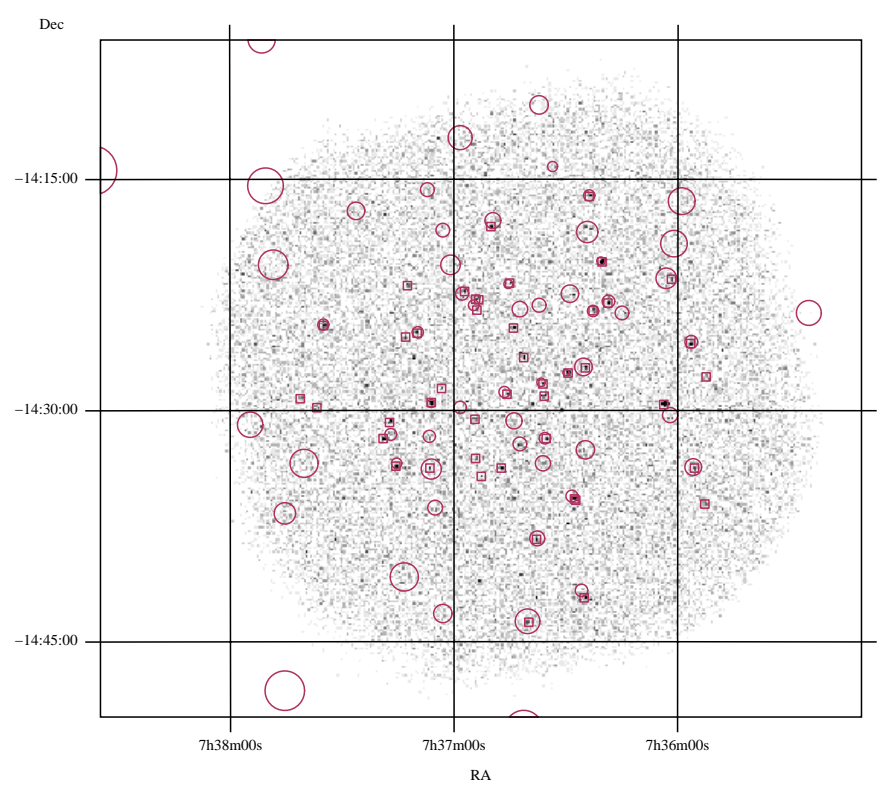

Fig. 2. X-ray image of an HRI $40 \mathrm{ks}$ observation pointed toward NGC 2422. The X-ray sources detected with the wavelet transform algorithm are identified by squares. Also indicated are positions of sources detected with the PSPC, with circle size denoting the PSPC error circle.

\subsection{X-ray flux and luminosity determination}

The typical number of counts inferred for PSPC detections ranges from 10 to 100 , with only six sources having more than 100 counts. With such low counting statistics, a spectral analysis based on parametric fitting is not feasible. In order to derive a conversion factor between count rate and luminosity in the $0.2-2.0 \mathrm{keV}$ energy range, we have assumed that the X-ray spectra can be described in terms of a Raymond-Smith (hereafter RS) single

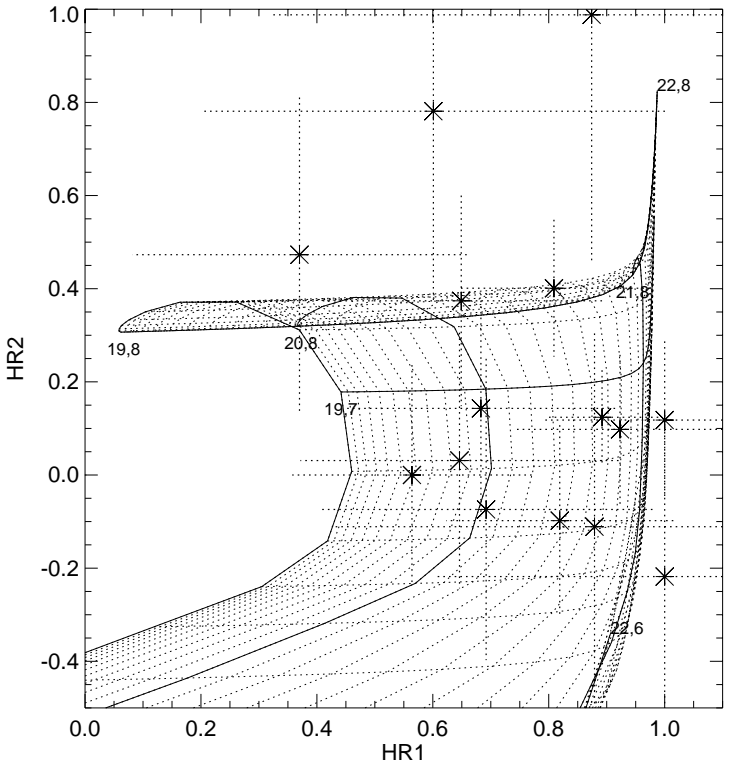

Fig. 3. Scatter plot of two PSPC hardness ratios of all the sources with signal-to-noise ratio larger than 6 , likely members of the cluster. Superimposed are the iso- $T$ (dashed line) and iso- $N_{\mathrm{H}}$ (solid line) curves derived using a single temperature RS model. The numbers labeling the curves indicate the logarithm of $N_{\mathrm{H}}$ and $T$ separated by a comma.

temperature model of thermal emission from optically thin plasma with solar abundances (Raymond \& Smith 1977). We have assumed that the hydrogen column density toward the cluster is $N_{\mathrm{H}}=2.9 \times 10^{20} \mathrm{~cm}^{-2}$, computed using the expression $N_{\mathrm{H}}=1.79 \times 10^{21} \times 3.1 \times E_{B-V}$ (Predehl \& Schmitt 1995) with $E_{B-V}=0.053$ (Nissen 1988). An average plasma temperature for stars of the cluster was derived from the analysis of standard hardness and color ratios calculated with the automated pipeline procedure described in Mackie et al. (1996). Figure 3 shows the scatter plot of the hardness ratios $H R 1$ and $H R 2^{1}$ for the PSPC sources with a signal-to-noise ratio larger than 6 , likely members of the cluster (cf. Sects. 3.2 and 3.4). Superimposed are the iso-temperature and iso- $N_{\mathrm{H}}$ curves calculated with a single temperature RS model. The figure shows a clustering of the sources in the region $T \sim 10^{6.8-7.0} \mathrm{~K}$ and $N_{\mathrm{H}}=10^{19.7-20.7} \mathrm{~cm}^{-2}$.

Based on this evidence we have adopted an average plasma temperature of $10^{6.8} \mathrm{~K}$ and have maintained the $N_{\mathrm{H}}$ value of $2.9 \times 10^{20} \mathrm{~cm}^{-2}$. With these choices the conversion factor between PSPC count rate in the $0.2-$ $2.0 \mathrm{keV}$ and the unabsorbed flux in the same band is $1.1 \times 10^{-11} \mathrm{erg} \mathrm{cm}^{-2} \mathrm{cnt}^{-1}$, while the corresponding conversion factor for the HRI is $2.7 \times 10^{-11} \mathrm{erg} \mathrm{cm}^{-2} \mathrm{cnt}^{-1}$. The uncertainties in $T$ and $N_{\mathrm{H}}$ introduce a relative uncertainty of $\$ 20 \%$ in the derived conversion factors for both PSPC and HRI.

1 The hardness ratios are defined as $H R 1=(H 1-S 1) /(H 1+$ $S 1)$ and $H R 2=(H 2-S 2) /(H 2+S 2)$, where the energy bands include the following PSPC channels, respectively: $S 1=3-10$ $(0.1-0.4 \mathrm{keV}), H 1=13-30(0.5-2.0 \mathrm{keV}), S 2=13-18(0.5-$ $0.9 \mathrm{keV})$ and $H 2=22-30(1.1-2.0 \mathrm{keV})$. 


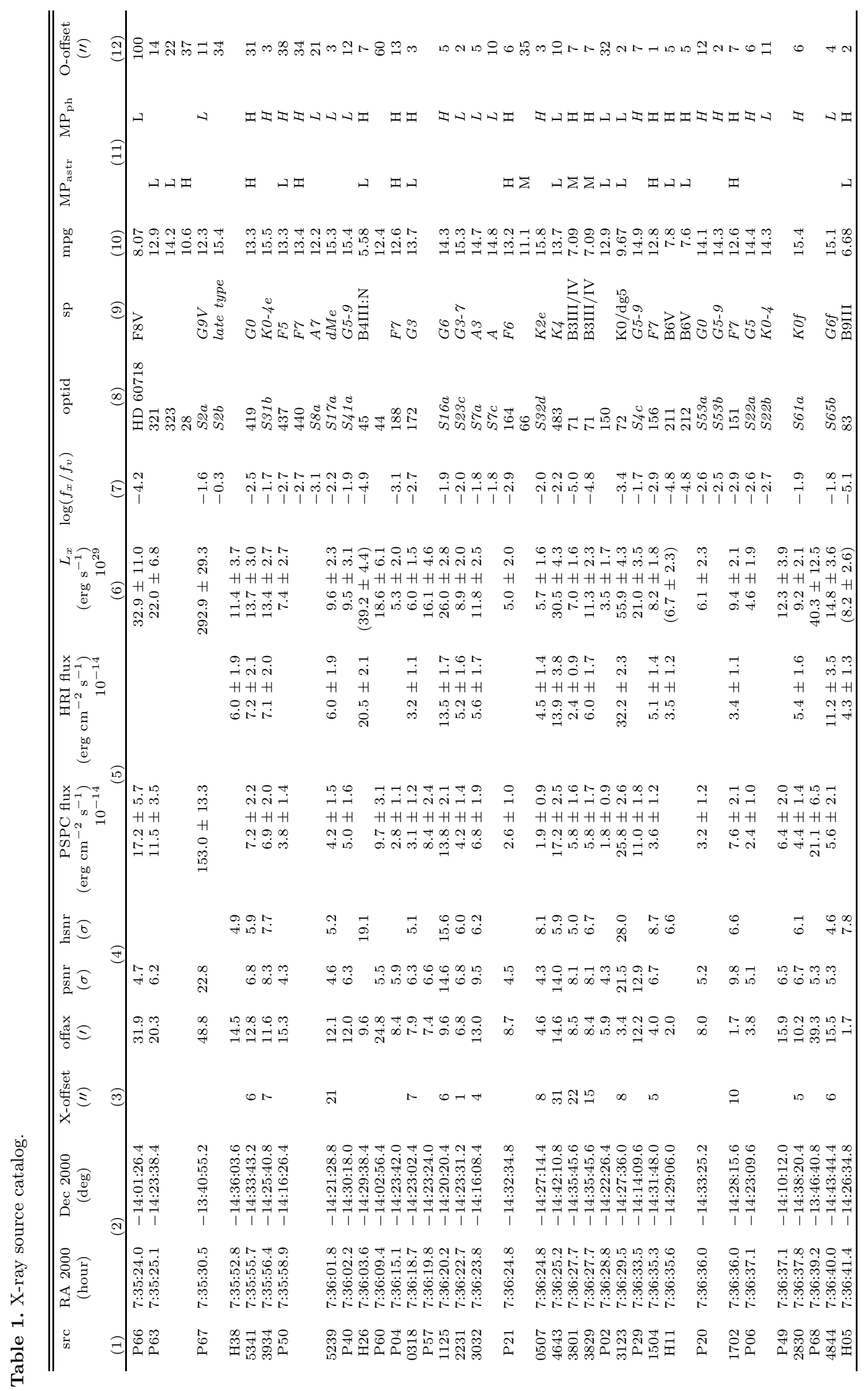




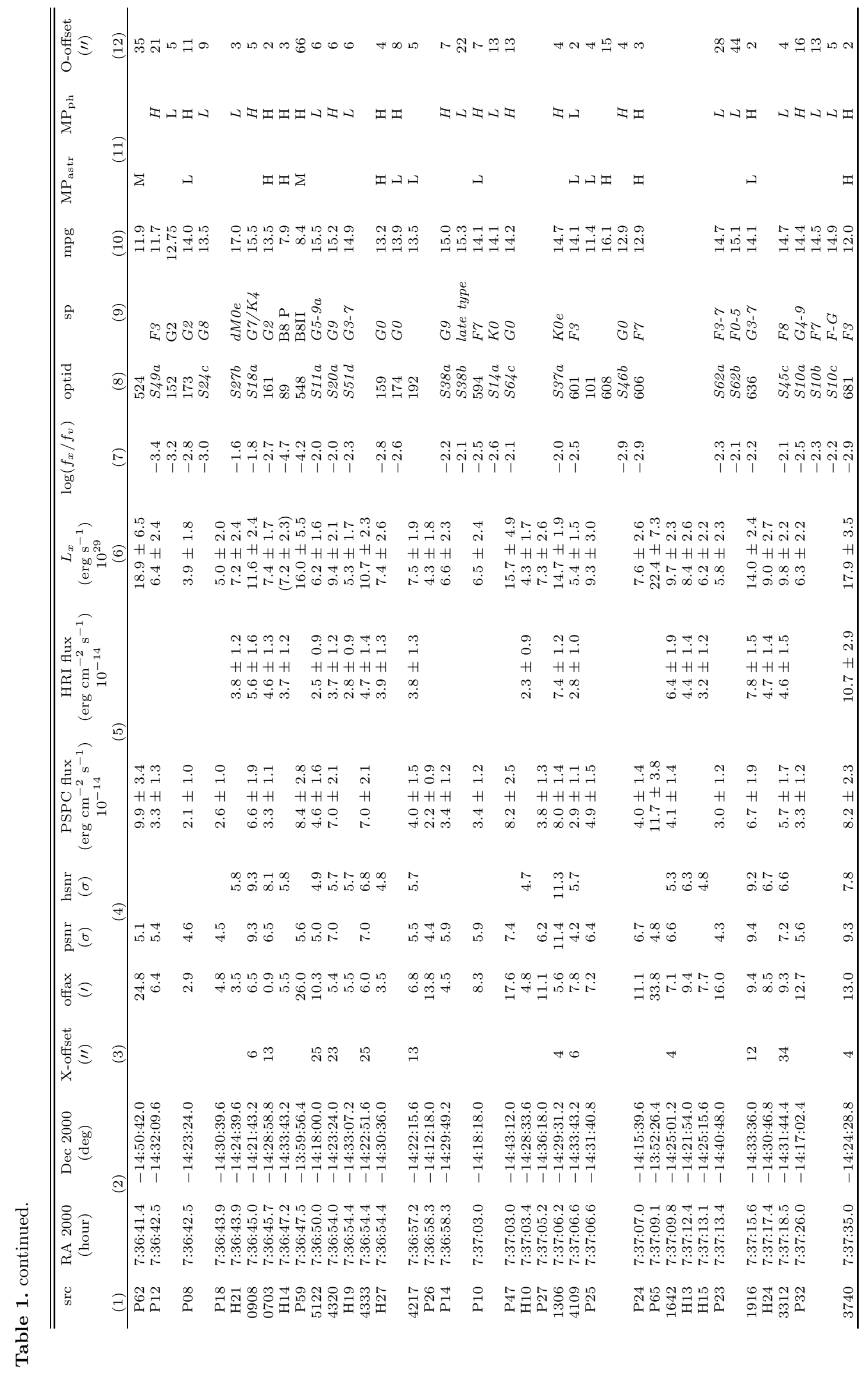


Table 1. continued.

\begin{tabular}{|c|c|c|c|c|c|c|c|c|c|c|c|c|c|c|c|c|}
\hline src & $\begin{array}{c}\text { RA } 2000 \\
\text { (hour) }\end{array}$ & $\begin{array}{l}\begin{array}{c}\text { Dec } 2000 \\
(\mathrm{deg})\end{array} \\
(2)\end{array}$ & $\begin{array}{c}\text { X-offset } \\
(\text { (II) } \\
(3)\end{array}$ & $\begin{array}{c}\text { offax } \\
(\prime)\end{array}$ & $\begin{array}{c}\operatorname{psnr} \\
(\sigma) \\
(\end{array}$ & $\begin{array}{c}\mathrm{hsnr} \\
(\sigma)\end{array}$ & $\begin{array}{c}\text { PSPC flux } \\
\left(\mathrm{erg} \mathrm{cm}^{-2} \mathrm{~s}^{-1} \text { ) }\right. \\
10^{-14}\end{array}$ & $\begin{array}{c}\text { HRI flux } \\
\left(\mathrm{erg} \mathrm{cm}^{-2} \mathrm{~s}^{-1}\right) \\
10^{-14} \\
5)\end{array}$ & $\begin{array}{c}L_{x} \\
\left(\mathrm{erg} \mathrm{s}^{-1}\right) \\
10^{29} \\
(6)\end{array}$ & $\begin{array}{c}\log \left(f_{x} / f_{v}\right) \\
(7)\end{array}$ & $\begin{array}{c}\text { optid } \\
(8)\end{array}$ & $\begin{array}{l}\mathrm{sp} \\
\text { (9) }\end{array}$ & $\begin{array}{l}\text { mpg } \\
\text { (10) }\end{array}$ & $\mathrm{MP}_{\text {astr }}$ & $\mathrm{MP}_{\mathrm{ph}}$ & $\begin{array}{c}\begin{array}{c}\text { O-offset } \\
(\prime \prime)\end{array} \\
(12)\end{array}$ \\
\hline $\mathrm{H} 37$ & $7: 37: 36.8$ & $-14: 29: 49.2$ & & 13.0 & & 4.7 & & $6.1 \pm 2.0$ & $11.8 \pm 3.8$ & & & & & & & \\
\hline P71 & $7: 37: 44.0$ & $-13: 40: 26.4$ & & 47.4 & 7.4 & & $31.1 \pm 7.8$ & & $59.4 \pm 15.2$ & & & & & & & \\
\hline P58 & $7: 37: 45.5$ & $-14: 36: 39.6$ & & 17.4 & 6.1 & & $7.9 \pm 2.5$ & & $15.1 \pm 4.9$ & -1.5 & $\begin{array}{l}S 60 a \\
S 60 b\end{array}$ & $\begin{array}{l}\text { G3-7 } \\
d M e\end{array}$ & $\begin{array}{l}15.7 \\
15.0\end{array}$ & & $L$ & $\begin{array}{l}13 \\
18\end{array}$ \\
\hline P64 & $7: 37: 45.5$ & $-14: 48: 10.8$ & & 26.1 & 5.2 & & $11.8 \pm 3.7$ & & $22.7 \pm 7.1$ & & 126 & & $\begin{array}{l}10.0 \\
10.8\end{array}$ & M & & $\begin{array}{l}1.1 \\
1.1\end{array}$ \\
\hline P61 & $7: 37: 51.6$ & $-14: 05: 56.4$ & & 25.1 & 7.5 & & $13.7 \pm$ & & $26.2 \pm 7.3$ & & 127 & & 10.5 & $\mathrm{H}$ & & 12 \\
\hline P44 & $7: 37: 54.5$ & $-14: 30: 54.0$ & & 16.7 & 4.9 & & $4.6 \pm 1.7$ & & $8.9 \pm 3.4$ & -2.8 & 719 & F7 & 12.9 & $\mathrm{H}$ & $\mathrm{H}$ & 20 \\
\hline P55 & $7: 38: 37.0$ & $-14: 14: 24.0$ & & 28.6 & 4.7 & & $4.4 \pm 1.6$ & & $8.4 \pm 3.1$ & & 765 & & 12.8 & $\mathrm{~L}$ & & 45 \\
\hline P69 & $7: 38: 43.8$ & $-14: 00: 10.8$ & & 38.0 & 9.4 & & $36.0 \pm 6.6$ & & $68.9+13.1$ & & 766 & & 12.6 & $\mathrm{~L}$ & & 96 \\
\hline
\end{tabular}

1) We have used the notation P $x x$ and H $x x$, where $x x$ are two digits, for the PSPC-only and HRI-only X-ray sources, and four digits for sources detected both in PSPC and HRI, with the first two digits giving PSPC source number.

(2) For detections in both the HRI and PSPC, we report HRI coordinates.

(3) Distance between the PSPC and HRI matching sources (arcsec).

(4) PSPC and HRI source detection probability expressed as the number of standard deviations of a Gaussian distribution.

(5) Unabsorbed X-ray flux $[0.2-2.0 \mathrm{keV}]$ and error. The corresponding measured count rates can be derived using the adopted values of the conversion factors, namely $1.1 \times$ $10^{-11} \mathrm{erg} \mathrm{cm}^{-2} \mathrm{cnt}^{-1}$, and $2.7 \times 10^{-11} \mathrm{erg} \mathrm{cm}^{-2} \mathrm{cnt}^{-1}$, for the PSPC and the HRI, respectively.

(6) X-ray luminosity [0.2-2.0 keV] and error, assuming a cluster distance of $470 \pm 10 \mathrm{pc}$ (Rojo Arellano et al. 1997). The adopted flux is a weighted average of PSPC and HRI values when both are available. Parenthesized values indicate possible UV contamination from hot stars near the X-ray position (cf. Sect. 4.1).

(7) Based on a typical uncertainty of 0.5 magnitudes on the apparent visual magnitudes (corresponding to an uncertainty of $0.5 / 2.5$ in log $(f v)$ ), and the quoted uncertainties on the X-ray fluxes, we estimate an uncertainty on $\log (f x / f v)$ of the order of 0.4 .

(8) The optical identification number is from the literature-based optical catalog, while the counterparts observed in the optical La Silla run are identified by a sequence number reported in italics prefixed by a capital S.

(9) Spectral types in italics are derived from the spectroscopic observations conducted at the ESO $1.50 \mathrm{~m}$ telescope in La Silla. An "e", "f", or "a" following the spectral type indicates that the spectroscopic observation shows the $\mathrm{H} \alpha$ line in emission (e), filled in (f) or in deep absorption (a).

(10) The photographic magnitude of the optical counterparts from our observations at ESO (optid starting with a capital S), have a typical uncertainty of 0.5 magnitudes. For the optical counterparts belonging to the literature-based optical catalog the photographic magnitude are from Ishmukhamedov (1967) that quotes a typical uncertainty of 0.2 magnitudes.

(11) Astrometric and photometric membership flags (cf. Sects. 3.2 and 3.4)

(12) Offset between X-ray source centroid and optical counterpart position. 


\section{The optical sample}

\subsection{An NGC 2422 literature-based optical catalog}

Mermilliod (1979) has compiled a cross correlation table between the numbering systems of various optical catalogs of stars in the region of NGC 2422, namely, in chronological order: Zug (1933), Lyngå (1959), Hoag et al. (1961), Smyth \& Nandy (1962), van Schewick (1966), Ishmukhamedov (1967). This cross-correlation table lists 212 different objects. Mermilliod (1986) has compiled mean photometric data and spectral classification of stars in the Mermilliod (1979) list. We have adopted this catalog as a starting point for building a wider literature-based compilation of measured data of stars in the field of NGC 2422, including coordinates, proper motion and more recent photometric data when available. We have adopted the numbering system of Mermilliod (1979) for the 212 stars reported therein. Our final compilation includes position (J2000), photographic and visual magnitudes, $U B V$ photometry, spectral classification, and proper motion for the 212 objects in Mermilliod (1986) plus 564 additional stars from Ishmukhamedov (1967). In cases where the same stellar parameter is reported in multiple papers, we have chosen the measurement with lowest uncertainty.

\subsection{Astrometric membership determination}

Proper motions reported in Ishmukhamedov (1967) have been used to assign a membership probability to the stars in the literature-based optical catalog with a similar method used for other young open clusters like the Pleiades (Hambly et al. 1991). Note that this sample of stars can be considered nearly complete up to $m_{\mathrm{ph}}=15$ corresponding to $B-V=0.7$ (early G type) for cluster main sequence stars.

In the plot of the relative proper motion shown in Fig. 4, the cluster centroid (van Schewick 1966) has been marked with a plus sign. The two circles centered on the cluster centroid have radii of $s$ and $2 \times s$, respectively, where $s$ is the measurement uncertainty in the proper motion quoted by Ishmukhamedov (1967). In the same plot, the circle centered on the axis intersection has radius equal to the standard deviation of the field star distribution. Stars within region Ia have been assigned a high (H) membership probability (MP), stars within region IIa have been assigned medium (M) MP, and all stars outside these regions have been assigned a low (L) MP.

Regions $\mathrm{Ib}$ and IIb, shown in Fig. 4 as coarsely hatched, have been used to evaluate the number of expected non-member objects in the corresponding cluster regions (Ia, IIa).

Non-member contamination fractions have been calculated by dividing the number of objects in region $\mathrm{Ib}$ (IIb) by the number of objects in region Ia (IIa). We estimate a contamination of $41 \%$ and $72 \%$ for regions Ia and IIa, respectively. The contamination from non-members

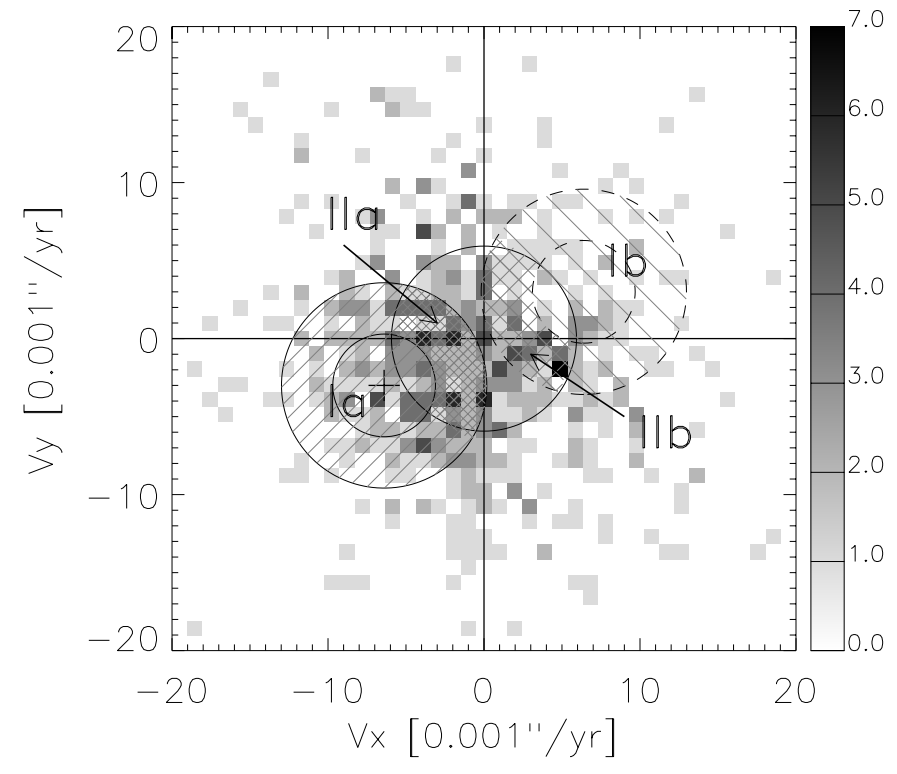

Fig. 4. Grey scale density map of the relative proper motion of the stars in the NGC 2422 region. The plus sign is the cluster centroid according to van Schewick (1966). Regions Ia, IIa, Ib, and IIb have been defined to assign a membership probability to the stars.

field objects is quite large both in the high and medium membership classes, and is mostly due to the large measurement uncertainty on the available proper motions and to the small offset of the cluster centroid with respect to the background star clump. In view of this evidence, we have avoided deriving general cluster properties using astrometric membership alone (cf. Sect. 4.3).

Since the cluster proper motion distribution is dominated by the measurement uncertainty and not by the intrinsic spread (this is generally true for young open clusters) an estimate of the completeness of the two regions Ia and IIa can be derived from a two dimensional Gaussian distribution. We estimate completeness of approximately $76 \%$ and $15 \%$ for regions Ia and IIa, respectively.

\subsection{The ESO $1.5 \mathrm{~m}$ telescope observations}

To achieve our goal of identifying optical counterparts for as many X-ray sources as possible, we found it necessary to carry out a detailed program of spectroscopic observations; existing data on NGC 2422 members were insufficient, particularly for late-type stars. Our spectroscopic program consisted of taking optical low resolution spectra for all possible counterparts in the field of each $\mathrm{X}$-ray source. The observations were carried out at the ESO $1.5 \mathrm{~m}$ telescope, using the B\&C spectrograph, at an effective 2 pixel resolution of about $4 \AA$, and with a coverage from $\sim 3700 \AA$ up to $\sim 7000 \AA$. The effective limiting magnitude was about 16 in the V band. For the NGC 2422 distance module of 8.48 , we are therefore able to investigate members down to late $\mathrm{G}$ /early $\mathrm{K}$.

In six nights of observations we covered $\sim 150$ possible counterparts, corresponding to a large fraction of sources 

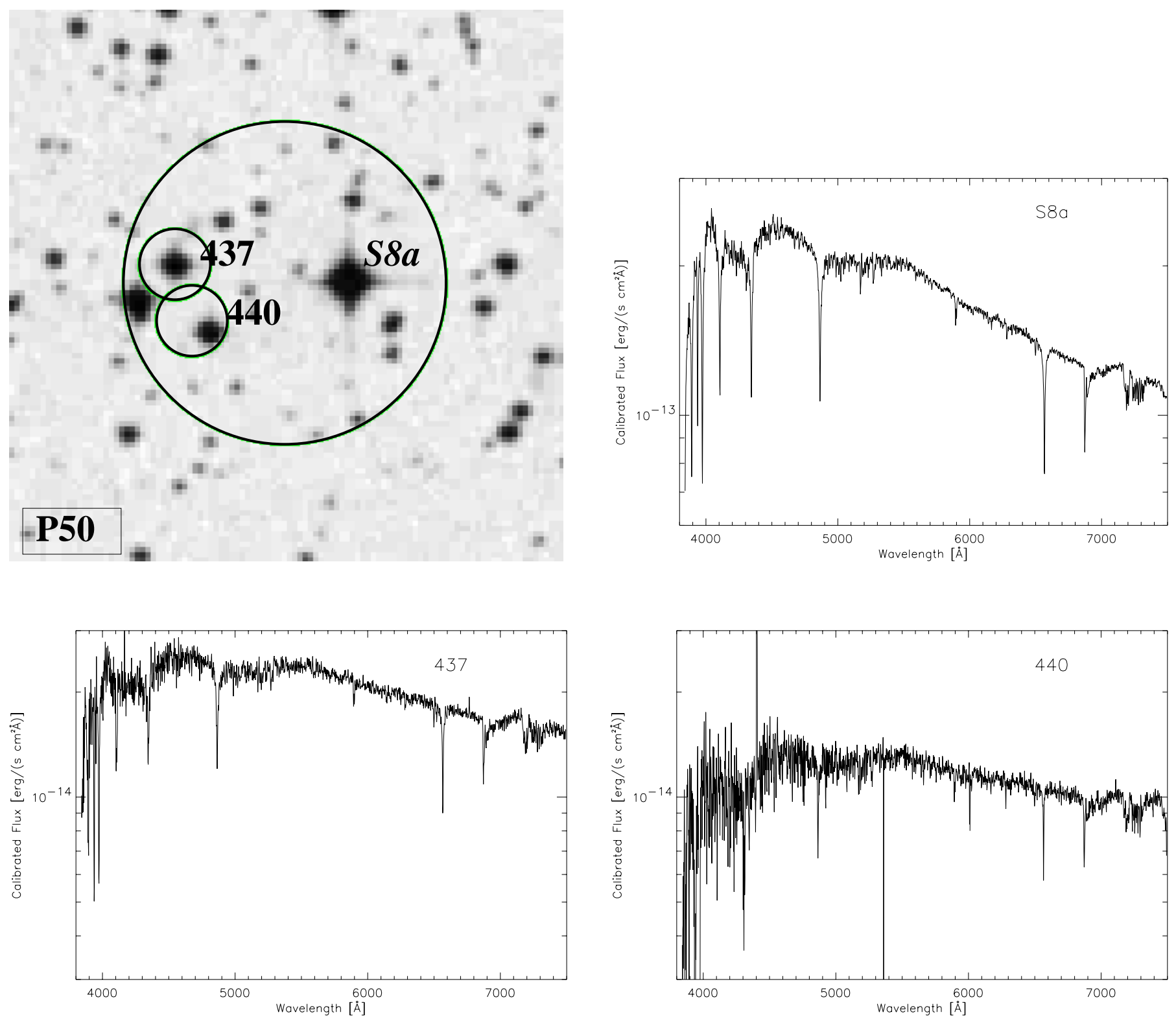

Fig. 5. Optical low dispersion spectra of three possible counterparts within the error circle (radius $=45^{\prime \prime}$ ) of the PSPC X-ray source 50. Three optical counterparts, S8a, 437, and 440, have been classified as A7, F5, and F7 spectral type, respectively. Both F stars likely contribute to the X-ray emission of the detected source, although other fainter optical sources might also contribute.

in our X-ray catalog. All optical objects in the PSPC error circles, within the limiting magnitude of about $m_{v}=16$, were observed (see e.g., Fig. 5), although some potential counterparts were missed due to bad weather at the end of our observing run. It should be noted that at this spectral resolution (and given the low sensitivity of the ESO $1.5 \mathrm{~m}$ CCD in the blue) no signs of stellar activity (i.e., Ca II emission cores) were visible in the majority of the counterparts, although some later type stars had (partially) filled in $\mathrm{H} \alpha$ lines. A few of the sources have as most likely counterparts $\mathrm{dMe} / \mathrm{dKe}$ stars.

Spectral types derived from the collected optical spectra are reported in Table 1 . Photographic magnitudes were derived from aperture photometry applied to the
Digitized Sky Survey plates, using the photometric calibrations given by Lasker et al. (1988). We estimate an uncertainty in the derived photographic magnitudes of \pm 0.5 mag. In Appendix A we show finding charts for all $\mathrm{X}$-ray sources.

\subsection{Photometric cluster membership}

For both the stars of the literature-based optical catalog and those we observed at the ESO $1.5 \mathrm{~m}$ telescope, we have assigned a photometric membership based on the distance from the zero age main sequence (ZAMS) of the cluster in the HR diagram, whenever the photographic magnitude and color index $B-V$ (and/or spectral type) 


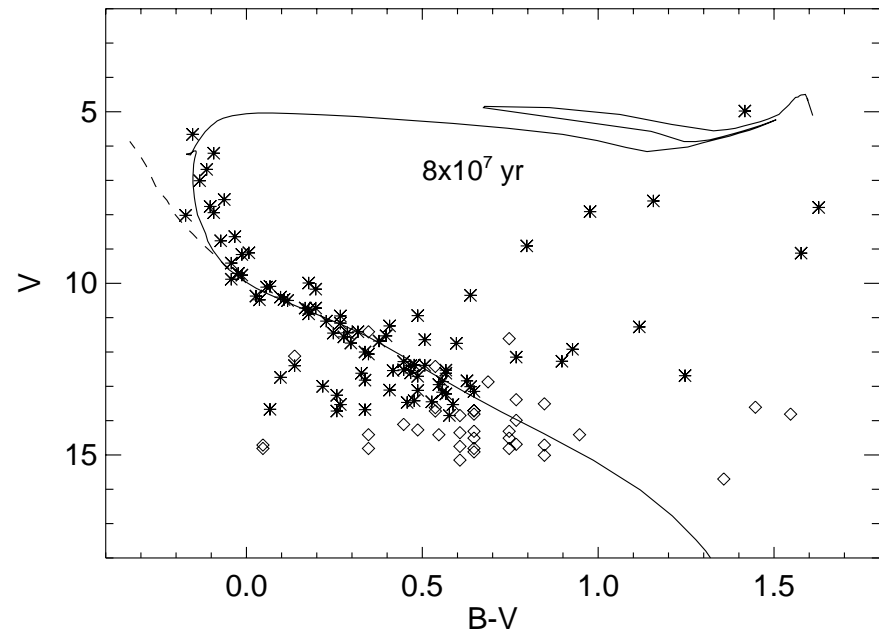

Fig. 6. HR diagram of the stars of the optical catalog with known $m_{v}$ and $B-V$ using $E(B-V)=0.053$ (Nissen 1988). The dashed line is the ZAMS of the cluster, and the solid line is the $8 \times 10^{7} \mathrm{yr}$ isochrone (Salasnich et al. 2000). Asterisks are stars from the literature-based optical catalog while diamonds are stars from our optical observation program at ESO.

is known. Figure 6 shows the HR diagram of the stars of the optical catalog with known visual magnitude and color index $B-V$ dereddened using $E(B-V)=0.053$ (Nissen $1988)$ together with the ZAMS of the cluster (dashed line) and the $8 \times 10^{7}$ yr isochrone derived from the data in Salasnich et al. (2000).

We have identified as having high photometric membership probability ("H" flag in Table 1) all stars within $0.8 \mathrm{mag}$ from the ZAMS of the cluster. In addition, for stars with photographic magnitude less than 10 (spectral types earlier than approximately A0) we also consider to have high membership probability those stars that are more luminous than the ZAMS by up to $2.5 \mathrm{mag}$; this accounts for the increase in luminosity of early type stars on the time scale of the life of the cluster, prior to the exhaustion of the hydrogen cores. Stars of known photographic magnitude and known color index $B-V$ and/or spectral type that are inconsistent with the above criteria have been assigned a low photometric membership probability ("L" flag in Table 1).

\section{Results}

\subsection{X-ray variability}

Many of the 37 PSPC sources with no HRI counterpart are detected outside the HRI central region of the FOV, while most of the 13 HRI sources with no PSPC counterpart are found in the central region of the FOV. In order to interpret these findings, we have constructed sensitivity threshold maps of the PSPC and HRI images using the Damiani et al. (1997a) wavelet transform algorithm.

The HRI is more sensitive than the PSPC in the $\sim 20^{\prime}$ diameter central region, while outside this region, the PSPC is more sensitive. Two sources (P29 and H26) were detected by only one of the two instruments at fluxes

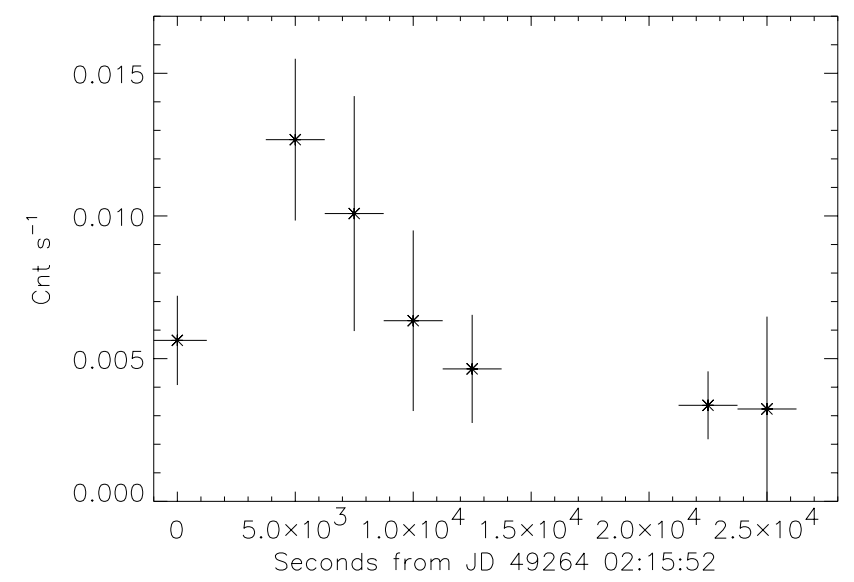

Fig. 7. Light curve of source P29, detected only by the PSPC. Its non-detection by the HRI is further evidence of variability.

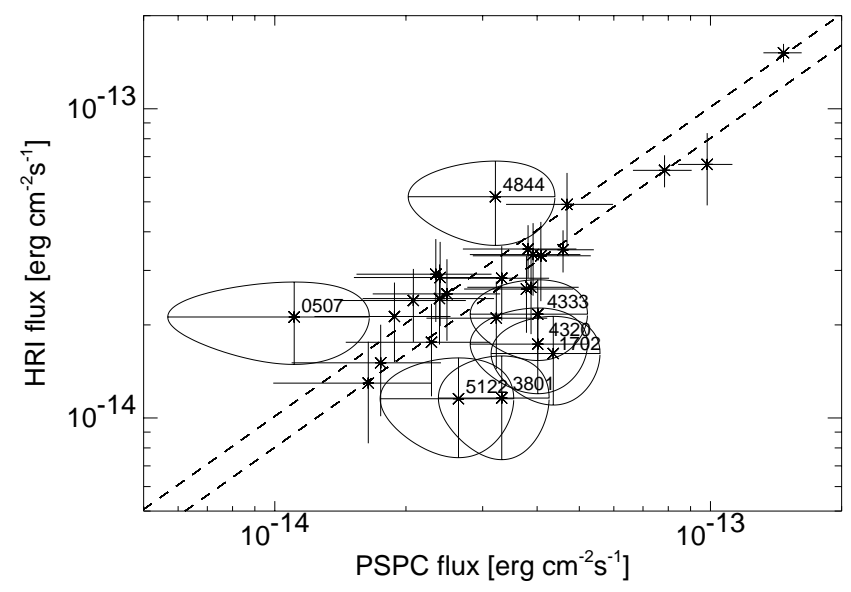

Fig. 8. Scatter plot of the $0.2-2.0 \mathrm{keV}$ X-ray flux measured with both the HRI and PSPC detectors. The superimposed error bars are the $1 \sigma$ uncertainties. For the 6 sources which are suspected to be variables, we have used an elliptical error region to better identify the allowed range for the fluxes. The two dashed lines define the range of X-ray flux uncertainty due to possible plasma temperature variation within the range $0.2-1.2 \mathrm{keV}$.

inconsistent with non-detection in the other, given its achieved sensitivity.

Source P29, a late G star detected only by the PSPC, shows a likely flare with a peak flux about 4 times the quiescent value and a decay time of the order of $10 \mathrm{ksec}$; its light curve is shown in Fig. 7. We have verified that the the HRI sensitivity threshold is consistent with a non detection of this source if it had a flux equal to its quiescent state in the PSPC light curve (count rate $=0.0035 \mathrm{cnt} / \mathrm{s}$ ). This evidence supports the indication of the timescale of this flare measured with the PSPC. Similar flares have been previously detected in G-type Pleiades stars, e.g. HZ 1136 (G8 type; Caillault \& Helfand 1985), and the stars HZ 345 and HZ 2147 (G8 and K0; Gagné et al. 1995).

Figure 8 is a scatter-plot of the $0.2-2.0 \mathrm{keV}$ flux of the sources detected by both detectors. The error bars are the $1 \sigma$ statistical uncertainties. The two dashed lines overplotted define the range of X-ray flux uncertainty due 
to plasma temperature variation within the range $k T=$ $0.2-1.2 \mathrm{keV}$.

This plot suggests seven candidates for long term variability: 0507, 5122, 3801, 4844, 4320, 4333, and 1702. PSPC source 43 matches HRI sources 20 and 33, and its measured flux is consistent with the flux sum of the two HRI sources. A similar argument applies to PSPC source 38 and its matching HRI sources 01 and 29. The excess PSPC flux of 1702 with respect to the HRI detection is likely due to the larger PSPC error circle which includes more than one source, as it is evident from the finding chart in Fig. A.1. None of the remaining three stars, namely 5122, 0507, and 4844, present evidence of flares in the light curves, and we have also verified that they have not peculiar hardness ratio values.

Given the sensitivity of the ROSAT HRI to ultraviolet radiation (Zombeck et al. 1997), we have examined all sources detected only with the HRI or with an HRI flux significantly higher than the PSPC flux, to ascertain whether any of the detected HRI counts could be ascribed to UV contamination from hot stars near the X-ray source positions. In order to evaluate the UV (1000-5500 A) contamination from hot stars we have adopted the calibration curves of the HRI UV sensitivity from Barbera et al. (2000), derived using the model of the HRI UV-Visible effective area described in Zombeck et al. (1997).

Five of 13 sources detected only with the HRI, namely H26, H11, H05, H14, and H19 have hot stars within their error circles. The HRI rate measured for source H26 can be entirely attributed to UV contamination from a positionally adjacent BIII star of $m_{V}=5.66$. Such UV contamination can also account for a significant fraction of the HRI rates of sources $\mathrm{H} 11, \mathrm{H} 05$, and $\mathrm{H} 14$. The UV contamination from the faint A type star $\left(m_{v}=10.2\right)$ in the error circle of source H19 is negligible.

None of the two sources with HRI flux $(0.2-2.0 \mathrm{keV})$ significantly larger than the PSPC flux, namely 0507, and 4844 (see Fig. 8) have a hot star within the error circle whose UV contamination can account for this discrepancy.

\subsection{Identifications}

We have spatially matched the literature-based optical catalog with our X-ray source positions using the X-ray error circles as described in Sect. 2.1. Thirty-three of $78 \mathrm{X}$ ray sources have been found with at least one counterpart in the literature-based optical catalog. Another 24 X-ray sources have at least one optical counterpart among the stars observed at the $1.5 \mathrm{~m}$ ESO telescope. For $18 \mathrm{X}$-ray sources with multiple optical counterparts we have used visible to X-ray flux ratios and distances between the $\mathrm{X}$-ray source and the optical counterparts as discriminating factors in the counterpart identification process.

The six right-most columns of Table 1 report the optical identification number of matched sources, spectral type when available, photographic magnitude, astrometric membership flag (Sect. 3.2), photometric membership flag (Sect. 3.4), distance between the X-ray source and the optical counterpart. For some individual sources, we report additional notes regarding the optical counterpart identification process in Appendix B.

\subsection{X-ray luminosity functions}

The literature-based optical catalog can be considered nearly complete up to $B-V=0.7$ corresponding to the early $\mathrm{G}$ type stars on the main sequence. Given this completeness, we have decided to construct maximum likelihood cumulative X-ray luminosity functions only for $\mathrm{F}$ $(0.35<B-V<0.55)$ and early-G $(0.55<B-V<0.7)$ type stars. In order to build representative luminosity functions of NGC 2422 we have selected only stars from the literature-based optical catalog with high photometric membership probability. The selected samples consist of $15 \mathrm{~F}$ type stars (3 detections, and 12 upper limits), and $10 \mathrm{G}$ type stars ( 5 detections, and 5 upper limits).

Figure 9 shows the maximum likelihood cumulative X-ray luminosity functions for NGC 2422 (solid line) compared to the luminosity functions of the Pleiades (dashed line) for the same spectral types (Micela et al. 1996). For both F- and G-type stars the luminosity functions of the two nearly coeval clusters are statistically indistinguishable as judged by application of a two sample test for censored data.

Because of its distance and the limited sensitivity of the observations, we have been able to probe only the high luminosity tails of the X-ray luminosity functions of NGC 2422. Deeper observations are needed to explore the median and the X-ray low luminosity population of the cluster (late-G, K and M stars). We recall that a few clusters such as Praesepe (Randich \& Schmitt 1995), NGC 6475 (Prosser et al. 1995) and Stock 2 (Sciortino et al. 2000) do not follow the same $L_{x}$-age relation as the bulk of the open clusters, in the sense that the median of their X-ray luminosity functions falls outside the relation derived from the other clusters, but their high luminosity tail are "typical". Our results confirm that the tails of the XLFs of NGC 2422 are consistent with those of the other clusters, but the limited sensitivity of our observations prevent us from drawing conclusions on the body of the distribution.

\section{Summary and conclusions}

We have presented results of an X-ray study of the young open cluster NGC 2422, based on ROSAT PSPC and HRI data. NGC 2422 is a southern open cluster at a distance of about $470 \mathrm{pc}$, with an age close to that of the Pleiades.

We have detected $78 \mathrm{X}$-ray sources in the two available observations, a 10-ks PSPC pointing and a 40-ks HRI one. Source position, count rate, and detection statistical significance have been derived with a detection algorithm based on wavelet transforms, particularly suited to detect faint sources in crowded fields. Among the 78 detected 

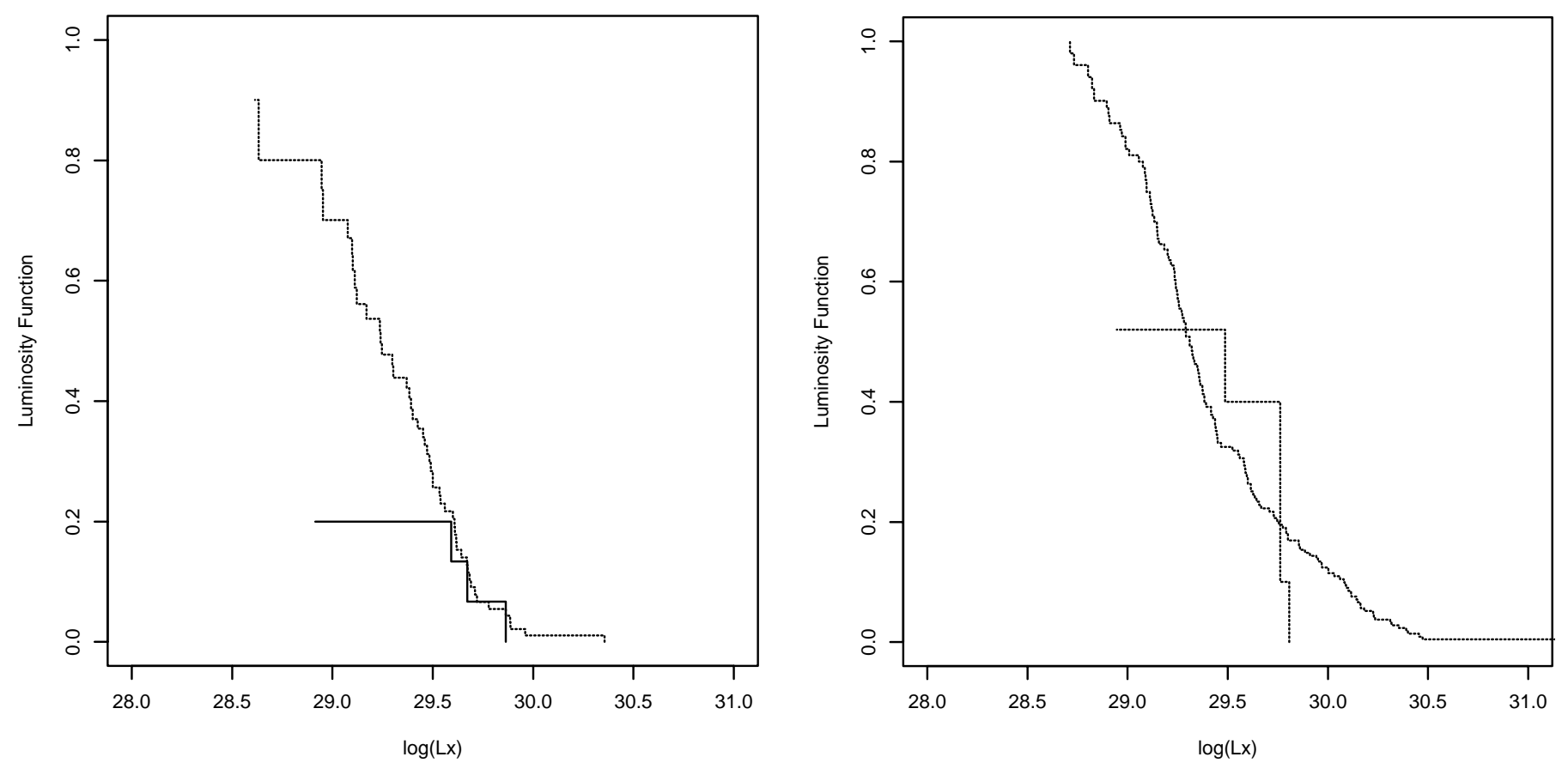

Fig. 9. Maximum likelihood cumulative X-ray luminosity functions for NGC 2422 (solid line) compared to the luminosity functions of the Pleiades (dashed line; Micela et al. 1996). The left panel is for F-type stars $(0.35<B-V<0.55)$, while the right panel is for early-G type stars $(0.55<B-V<0.7)$.

sources, 13 have been detected only with the HRI, and 37 only with the PSPC.

In cases where the PSPC and HRI fluxes of the same source were inconsistent, we have investigated possible effects due to source flaring and to the HRI "UV leak". Source P29, a late G type star detected only by the PSPC, underwent a flare with a peak flux about 4 times the quiescent value. The measured HRI rate of the source H26 can be entirely ascribed to the UV contamination from a BIII star within its error circle, and UV contamination can also explain the HRI rates of three other HRI detections.

Data from the literature and from our own optical observations have allowed us to find candidate optical counterparts for $62 \mathrm{X}$-ray sources, with more than $80 \%$ of these identifications being with late-type stars.

The number of sources with high membership probability counterparts (38 of 62 ) is in good agreement with expectations. In the Galactic plane we expect, on average, $26 \mathrm{X}$-ray sources with significance greater than $4.2 \sigma$ in a PSPC field of sensitivity similar to our PSPC observation (Pye et al. 1997; Sciortino et al. 1998). Since we detected 65 sources in the PSPC field, we expect that we have detected $\sim 40$ cluster members.

We have computed maximum likelihood cumulative $\mathrm{X}$-ray luminosity functions for $\mathrm{F}$ - $(0.35<B-V<0.55)$ and early-G $(0.55<B-V<0.7)$ type stars for which the literature-based optical catalog can be considered complete. The maximum likelihood X-ray luminosity functions of the $\mathrm{dF}$ and early $\mathrm{dG}$ type stars of this cluster are not statistically distinguishable from the high luminosity tails of the luminosity functions of the nearly coeval Pleiades cluster.
Acknowledgements. We acknowledge partial support by Agenzia Spaziale Italiana and Ministero dell'Istruzione dell'Università e della Ricerca (MB, FB, FD, GM, SS). F. Bocchino acknowledges an ESA Research Fellowship. FRH received partial support from NASA grant NAS5-4967 and NASA contract NAS8-38248. We also acknowledge use of the SIMBAD astronomical database of the Centre de Données astronomiques de Strasbourg. The Digitized Sky Surveys were produced at the Space Telescope Science Institute under U.S. Government grant NAG W-2166. The images of these surveys are based on photographic data obtained using the Oschin Schmidt Telescope on Palomar Mountain and the UK Schmidt Telescope. The plates were processed into the present compressed digital form with the permission of these institutions.

\section{Appendix A: Finding charts of the $X$-ray sources}

Figure A. 1 provides $3^{\prime} \times 3^{\prime}$ finding charts, based on the DSS plates, for all the detected X-ray sources. Superimposed are: a circle with radius equal to the X-ray source error circle (HRI when available) centered on the X-ray source position, circles with $10^{\prime \prime}$ radii centered onto the optical counterparts from the literature-based optical catalog, identification numbers for the optical counterparts from both the literature-based optical catalog, and our followup optical identification program (the identification numbers of these counterparts are prefixed by an $\mathrm{S}$ ). 


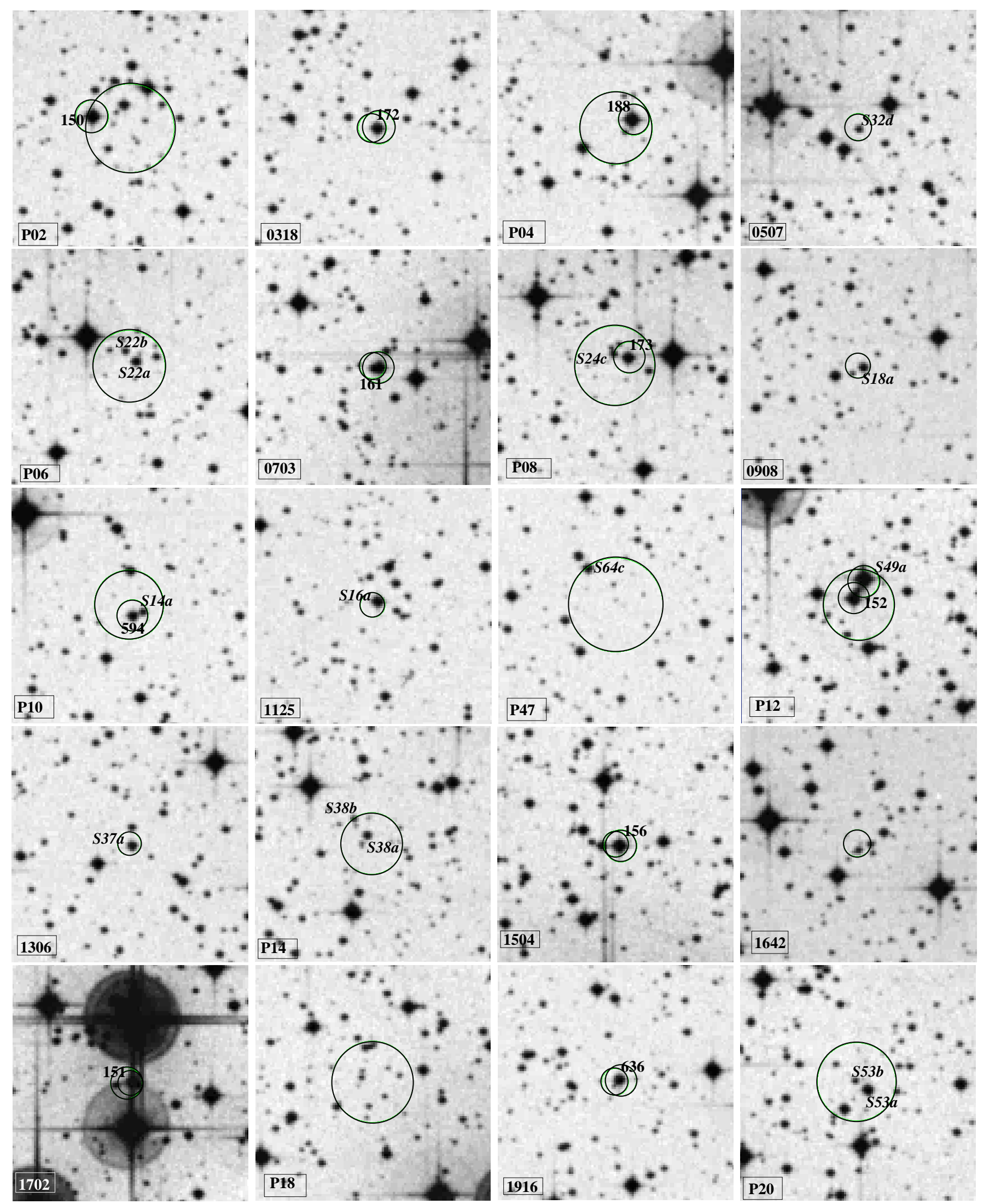

Fig. A.1. $3^{\prime} \times 3^{\prime}$ finding charts of the X-ray sources. Details on the overlays are discussed in Appendix A. 


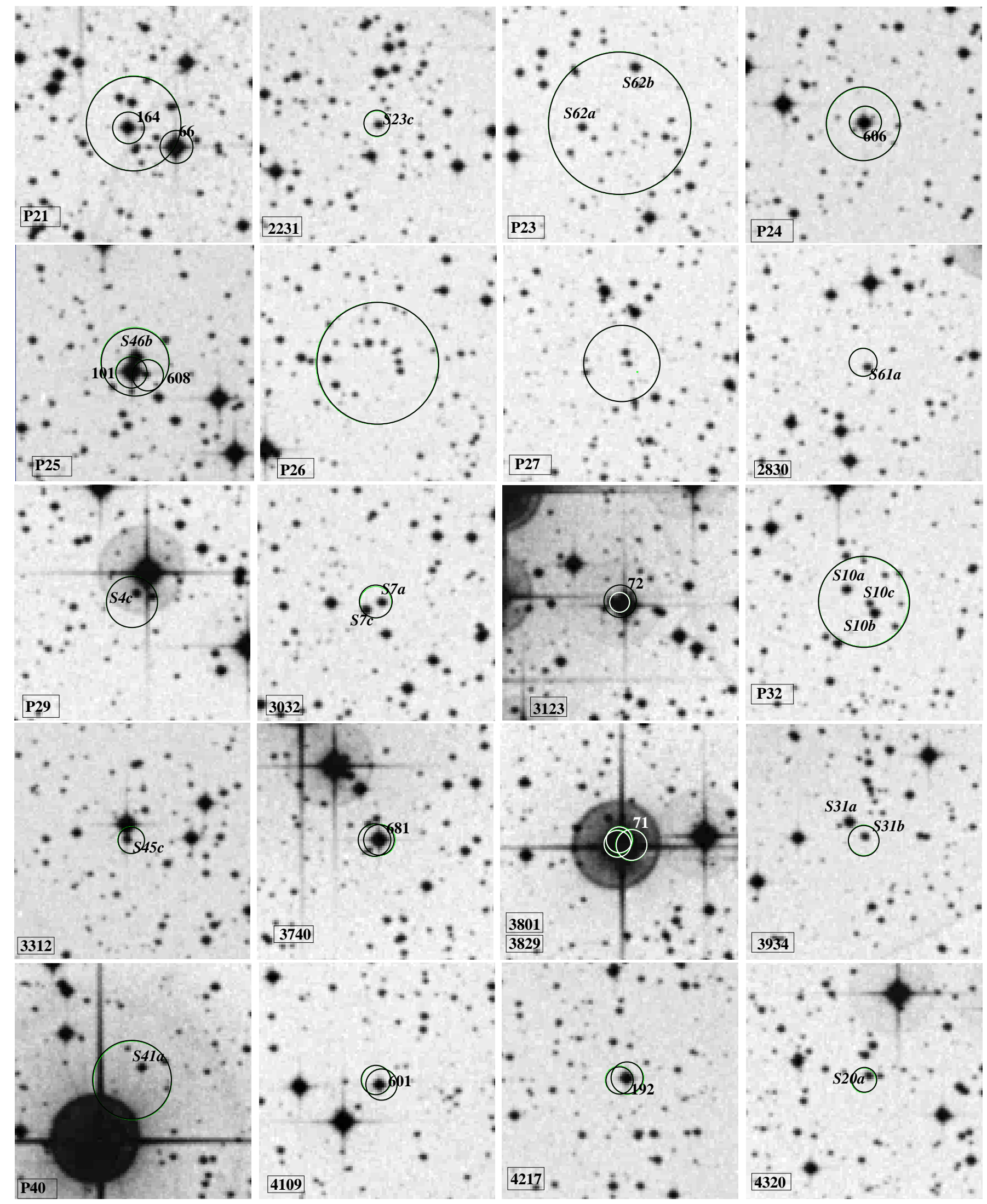

Fig. A.1. continued. 


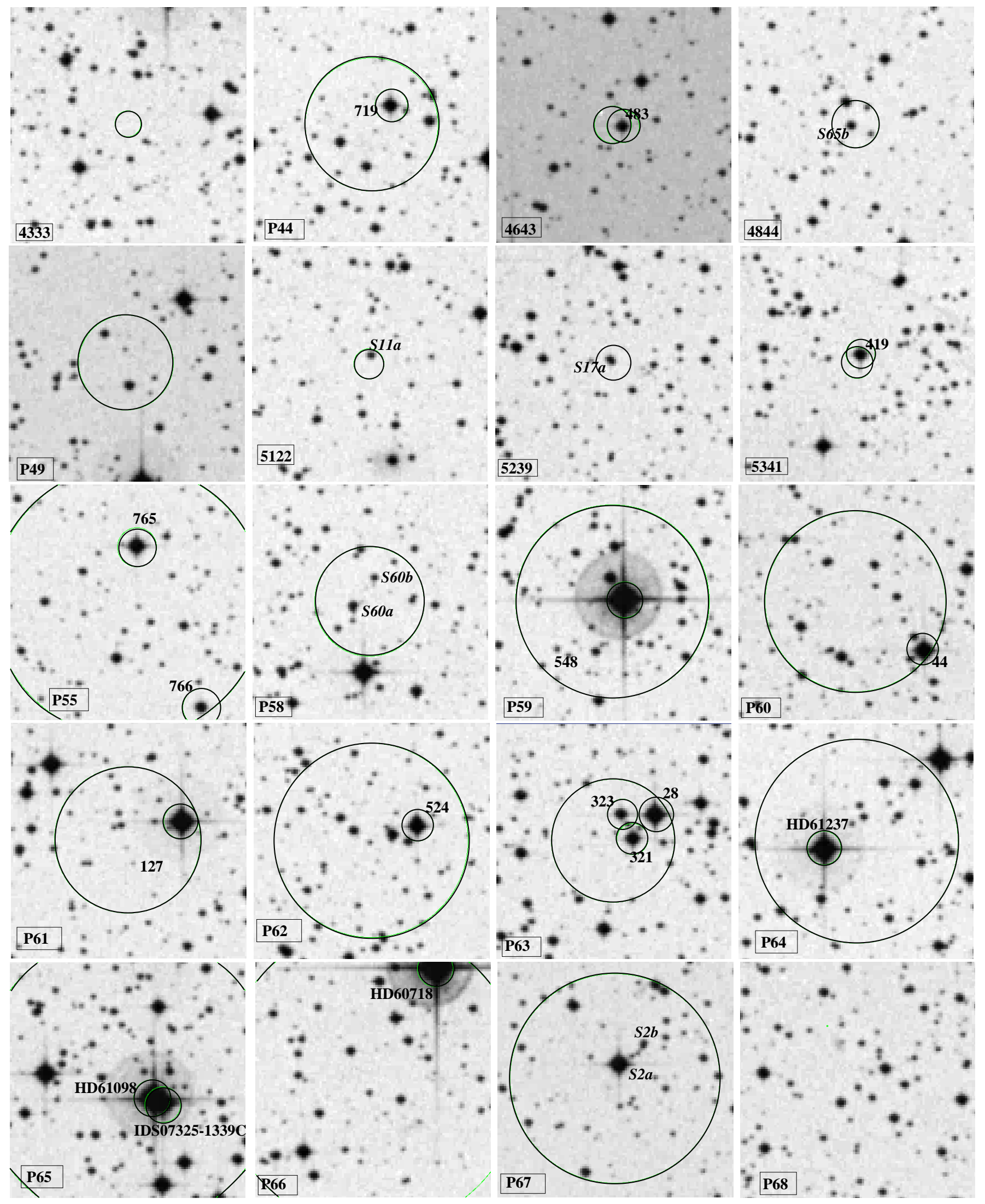

Fig. A.1. continued. 


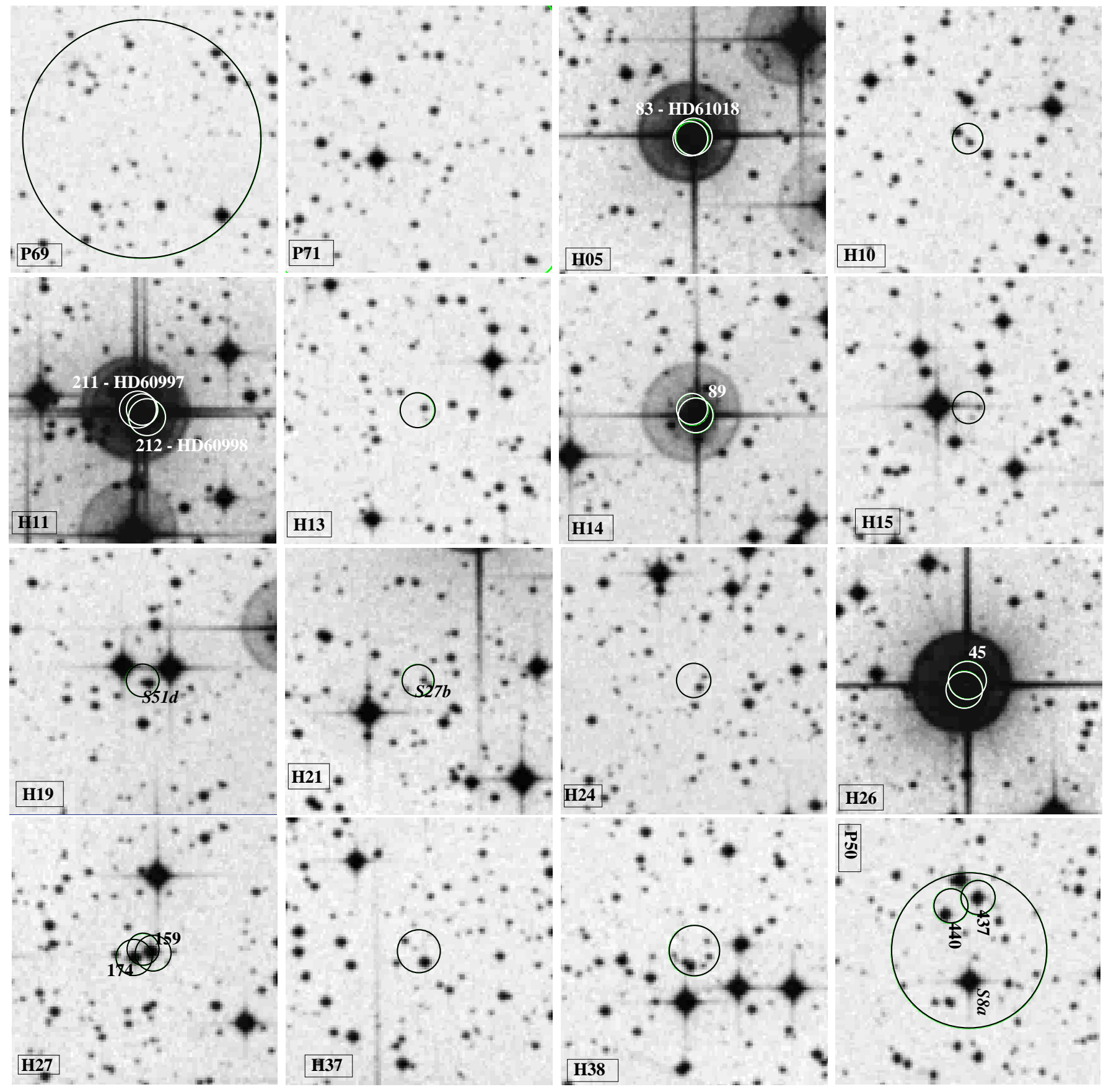

Fig. A.1. continued.

\section{Appendix B: Notes on individual sources}

$\mathrm{P} 67$ : the $f_{x} / f_{v}$ ratio is too high for the late type counterpart S2b. The G9 type star S2a has an acceptable value of $f_{x} / f_{v}$ and is also much closer to the X-ray source centroid, thus being the most likely counterpart.

P50: crowded field. 437 and 440 may both contribute to the detected count rate.

3032: the $f_{x} / f_{v}$ ratio are very high for the two A type stars positionally matching with the X-ray source. Neither is a likely counterpart.

P21: the star 164 (F6 type) is likely the counterpart of the $\mathrm{X}$-ray source; it is very close to the $\mathrm{X}$-ray source centroid and has an acceptable value of $f_{x} / f_{v}$. Star 164 is also very likely a cluster member.

P29: $\mathrm{S} 4 \mathrm{c}$ is a late $\mathrm{G}$ type star with $\mathrm{H} \alpha$ filled-in. Detected only by the PSPC, this source presents a likely flare with peak flux about 4 times the quiescent value and decay time of the order of $10 \mathrm{ks}$.

P20: S53a (G0 type star) and S53b (G5-9 type star) have acceptable values of $f_{x} / f_{v}$. S53b is coincident with the $\mathrm{X}$-ray centroid position.

P06: both S22a (G5 type star) and S22b (K0-4 type star) may both contribute to the detected count rate. 
P12: both S49a (F3 type star) and 152 (G2 type star) have acceptable values of $f_{x} / f_{v}$. 152, however, is much closer to the X-ray source centroid.

P08: S24c is a G8 type star with possible Ca II emission cores. Both S24c and 173 (G2 type star) likely contribute to the detected count rate.

0703: S36a (161 in the literature based optical catalog) is a G2 type star which looks elongated on the plate (possible faint companion).

H27: both 159 (G0 type star) and 174 (G0 type star) have an acceptable value of $f_{x} / f_{v}$ and likely both contribute to the detected count rate.

P14: both S38a (G9 type star) and S38b (late type star) have acceptable values of $f_{x} / f_{v}$. S38a is, however, much closer to the X-ray centroid position.

P10: S14a is a K0 type star with $\mathrm{H} \alpha$ filled in. S14a and 594 (F7 type star) both have acceptable values of $f_{x} / f_{v}$ and likely both contribute to the detected count rate.

P25: 101 and S46b (G0 type star) are both very close to the X-ray source centroid, and likely both contribute to the detected count rate.

P23: S62a is an F3-7 type star which could be composite. Both S62a (F3-7 type star) and S62b (F0-5 type star) have acceptable values of $f_{x} / f_{v}$ and likely both contribute to the detected count rate.

P32: S10a is a G4-9 type star with an $\mathrm{H} \alpha$ emission core. S10a, S10b (F7 type star), and S10c (F-G type star) have acceptable values of $f_{x} / f_{v}$, however, S10c is much closer to the position of the X-ray source centroid.

P58: S60a (G3-7 type star) and S60b (dMe type star) both have acceptable values of $f_{x} / f_{v}$ and likely both contribute to the detected count rate.

P71: one of the brightest X-ray sources in the field with a very soft spectrum $(H R=-0.4 \pm 0.1)$, it falls 47 arcmin offaxis. No optical counterpart is available within the $2^{\prime} \mathrm{X}$-ray source error circle radius both from the literature-based optical catalog and from the SIMBAD database of the Centre de Données astronomiques de Strasbourg.

P58: 766 is at the edge of the X-ray source error circle. 765 is closer to the position of the $\mathrm{X}$-ray source centroid but many other fainter stars in the X-ray source error circle may also contribute to the detected count rate.

\section{References}

Barbera, M., Micela, G., Collura, A., Murray, S., \& Zombeck, M. 2000, ApJ, 545, 449

Barrado y Navascués, D., Stauffer, J. R., \& Randich, S. 1998, ApJ, 506, 347

Bocchino, F., Barbera, M., \& Sciortino, S. 1998, A\&A, 129, 1

Caillault, J. P., \& Helfand, D. J. 1985, ApJ, 289, 279

Caillault, J. P. 1996, ASP Conf. Ser., 109, 325

Damiani, F., Maggio, A., Micela, G., \& Sciortino, S. 1997a, ApJ, 483, 350

Damiani, F., Maggio, A., Micela, G., \& Sciortino, S. 1997b, ApJ, 483, 370

David, L. P., Harden, F. R., Jr., Kearns, K. E., \& Zombeck M. V. 1995, U.S. ROSAT Science Data Center Calibration Memo
Favata, F., Micela, G., Sciortino, S., \& Morale, F. 1997, A\&A, 324, 998

Gagné, M., Caillault, J., \& Stauffer, J. R. 1995, ApJ, 450, 217

Hambly, N. C., Hawkins, M. R. S., \& Jameson, R. F. 1991, MNRAS, 253, 1

Harris, G. L. H. 1976, ApJS, 30, 451

Hasinger, G., Boese, G., Predhel, P., et al. 1993, MPE/OGIP Calibration Memo CAL/ROS/93-015

Hoag, A. A., Johnson, H. L., Iriarte, B., et al. 1961, Publications of the U.S. Naval Observatory Second Ser., 17,345

Holland, K., Jameson, R. F., Hodgkin, S., Davies, M. B., \& Pinfield, D. 2000, MNRAS, 319, 956

Ishmukhamedov, Kh. Z. 1967, TSIRK. Astr. Obs. Tashkent No. 346

Jeffries, R. D. 1999, ASP Conf. Ser., 158, 75

Lasker, B. M., Sturch, C.R., Lopez, C., et al. 1988, ApJS, 68, L1

Lasker, B. M., Sturch, C. R., McLean, B. J., et al. 1990, AJ, 99, 2019

Lyngå, G. 1959, Arkiv for Astronomi, 2, 379

Mackie, G., Fabbiano, A. G., Barbera, M., et al. 1996, ASP Conf. Ser., 101, 179

Mermilliod, J. 1979, A\&AS, 36, 163

Mermilliod, J. C. 1986, Bulletin d'Information du Centre de Données Stellaires, 31, 175

Micela, G., Sciortino, S., Kashyap, V., Harden, F. R., Jr., \& Rosner, R. 1996, ApJS, 102, 75

Morale, F., Micela, G., Favata, F., \& Sciortino, S. 1996, A\&A, 119, 403.

Nissen, P. E. 1988, A\&A, 199, 146

Pfeffermann, E., Briel, U.G., Hippmann, H., et al. 1987, Proc. SPIE, 733, 519

Predehl, P., \& Schmitt, J. H. M. M. 1995, A\&A, 293, 889

Prosser, C. F., Stauffer, J. R., Caillault, J. P., et al. 1995, AJ, 110,1229

Pye, J. P., Morley, J. E., Warwick, R. S., et al. 1997, Mem. S. A. It., 68, 1089

Randich, S. 1997, Mem. S. A. It., 68, 971

Randich, S., Schmitt, J. H. M. M. 1995, A\&A, 298, 115

Raymond, J. C., \& Smith, B. W. 1977, ApJS, 35, 419

Robichon, N., Arenou, F., Mermilliod, J., \& Turon, C. 1999, A\&A, 345, 471

Rojo Arellano, E., Pena, J. H., \& Gonzalez, D. 1997, A\&AS, 123,25

Salasnich, B., Girardi, L., Weiss, A., \& Chiosi, C. 2000, A\&A, 361,1023

Sciortino, S., Damiani, F., Favata, F., Micela, G., \& Pye, J. 1998, Astron. Nachr., 319, 108

Sciortino, S., Micela, G., Favata, F., Spagna, A., \& Lattanzi, M. G. 2000, A\&A, 357, 460

Shobbrook, R. R. 1984, MNRAS, 211, 659

Smyth, M. J., \& Nandy, K. 1962, Publications of the Royal Observatory of Edinburgh, 3, 24

van Rensbergen, W., Hammerschlag-Hensberge, G., \& van den Heuvel, E. P. J. 1978, A\&A, 64, 131

van Schewick, H. 1966, Veroeffentlichungen des Astronomisches Institute der Universitaet Bonn, 74, 1

Zombeck, M. V., David, L. P., Hardnen, F. R. Jr., \& Kearns, K. 1995, Proc. SPIE, 2518, 304

Zombeck, M. V., Barbera, M., Collura, A., \& Murray, S. S. 1997, ApJ, 487, L69

Zug, R. S. 1933, Lick Obs. Bull., 454, 119 